\title{
Pain Perception in Neurodevelopmental Animal Models of Schizophrenia
}

\author{
M. FRANĚK ${ }^{1}$, S. VACULÍN ${ }^{1}$, A. YAMAMOTOVÁ ${ }^{1}$, F. ŠŤASTNÝ ${ }^{2}$, V. BUBENÍKOVÁ- \\ VALEŠOVÁ ${ }^{2}$, R. ROKYTA ${ }^{1}$
}

${ }^{1}$ Charles University in Prague, Third Faculty of Medicine, Department of Normal, Pathological and Clinical Physiology, Prague, Czech Republic, ${ }^{2}$ Prague Psychiatric Center affiliated with the Charles University in Prague, Prague, Czech Republic

Received February 17, 2009

Accepted January 18, 2010

On-line April 20, 2010

\section{Summary}

Animal models are important for the investigation of mechanisms and therapeutic approaches in various human diseases, including schizophrenia. Recently, two neurodevelopmental rat models of this psychosis were developed based upon the use of subunit selective $N$-methyl-D-aspartate receptor agonists - quinolinic acid (QUIN) and $N$-acetyl-aspartyl-glutamate (NAAG). The aim of this study was to evaluate pain perception in these models. QUIN or NAAG was infused into lateral cerebral ventricles neonatally. In the adulthood, the pain perception was examined. The rats with neonatal brain lesions did not show any significant differences in acute mechanical nociception and in formalin test compared to controls. However, the neonatally lesioned rats exhibited significantly higher pain thresholds in thermal nociception. Increased levels of mechanical hyperalgesia, accompanying the sciatic nerve constriction (neuropathic pain), were also observed in lesioned rats. Although hyperalgesia was more pronounced in QUIN-treated animals, the number of c-Fos-immunoreactive neurons of the lumbar spinal cord was similar in experimental and control rats. We conclude that neonatal brain lesions attenuated the thermal perception in both nociceptive and neuropathic pain whereas mechanical pain was increased in the model of neuropathic pain only. Thus, nociceptive and neuropathic pain belongs - in addition to behavioral changes among the parameters which are affected in described animal models of schizophrenia.

\section{Key words}

Quinolinic acid • N-acetyl-aspartyl-glutamate $\bullet$ Neuropathic pain • Rat

\section{Corresponding author}

Miloslav Franek, Charles University in Prague, Third Medical Faculty, Department of Normal, Pathological and Clinical Physiology, Ke Karlovu 4, 12000 Prague 2, Czech Republic. Fax: +420 224916 896. E-mail: franek@lf3.cuni.cz

\section{Introduction}

Schizophrenia is a neurodevelopmental disorder which afflicts about $1 \%$ of the human population worldwide. The etiology and pathogenesis of this psychosis is complex but involves the interplay of polygenic influences and environmental risk factors operating on brain maturation processes. Different theories as to the cause of schizophrenia and the heterogeneity of clinical symptoms have made it difficult to develop a valid animal model. A number of limited animal models have been developed to explore various theories of etiology, progression and treatment of this psychosis (Marcotte et al. 2001). Neonatal excitotoxic disconnection of the fronto-temporo-hippocampal complex may represent a heuristic model of schizophrenia-like behavior (Bubeníková-Valešová et al. 2006, Lipska 2004, Št'astný et al. 2005). Two of these models have been based on the neonatal intracerebroventricular (i.c.v.) infusion of substances, which activate $N$-methyl-D-aspartate receptor (NMDA$\mathrm{R})$, when overproduced by activated microglia quinolinic acid (QUIN) (Heyes et al. 1996, Št'astný et al. 2005) and $N$-acetyl-aspartyl-glutamate (NAAG) 
(Bubeníková-Valešová et al. 2006, Passani et al. 1998). Similarities in behavioral changes and changes in social interactions were observed between these models and a genetic model of reduced NMDA-R function (Duncan et al. 2004).

It is well known that QUIN and NAAG have also antinociceptive effects on the pain experience (Heyliger et al. 1998, Yamamoto et al. 2004). The effect might be related to a hypofunction of NMDA-R in rat lumbar spinal cord (Boyce et al. 1999). The individuals with schizophrenia (and their relatives) (Hooley and Delgado 2001) are significantly less sensitive to direct painful stimuli (Dworkin 1994, Fishbain 1982, Kudoh et al. 2000, Rosenthal et al. 1990), but their subjective evaluation of the stimulus is similar to that of healthy control subjects (Blumensohn et al. 2002). It may reflect a dysfunction of glutamatergic system in these patients, which affects the transmission of the noxious stimuli.

To solve this problem we can use animal models although there are difficulties to evaluate the typical psychotic symptoms (hallucinations, lack of motivation, social withdrawal, attention deficits) in animals. However, it is important to know that the nociceptive profile of these models can help to interpret the clinical results obtained in nociceptive tests owing to a validity of the neurodevelopmental animal models of schizophrenia. The changes of pain perception in animal models are not homogenous and depend on used model (Al Amin et al. 2004, Gao et al. 2004, Schneider and Przewlocki 2005). The purpose of our study was to describe nociceptive profiles of adult animals (which had been neonatally treated with icv QUIN or NAAG) with neonatal brain lesions induced by QUIN or NAAG.

\section{Methods}

Animals

We have been using Wistar:Hann rats (SPF, BioTest, Konárovice Czech Republic) throughout the experiments. The litters were rearranged so that each contained eight males and two females. Mothers with their pups were housed in cages having free access to food and water. Animals were kept at $22 \pm 2{ }^{\circ} \mathrm{C}$ (relative humidity was 40-70\%) under 12-h light/12-h dark cycle. All experiments were approved by the Committee for Animal Care and Use of the $3^{\text {rd }}$ Faculty of Medicine (Charles University, Prague) and conducted in accordance with the ethical guidelines of the International Association for the Study of Pain (Zimmermann 1983).

\section{Neonatal brain lesions}

Wistar male pups on postnatal day 12 (PND 12) were anesthetized by ethylether, fixed in a stereotaxic apparatus (Stoelting, Wood Dale, IL) using ear and tooth bars. Quinolinic acid (QUIN group) or $N$-acetyl-aspartylglutamate (NAAG group) was slowly infused i.c.v. in a dose of $250 \quad \mathrm{nmol} / 0.25 \mu \mathrm{l}$ buffered isotonic saline/ventricle; one injection was given into each lateral cerebral ventricle by a $1 \mu$ l Hamilton syringe equipped with 26S needle with coordinates previously used (Bubeníková-Valešová et al. 2006, Lisý et al. 1994, Št'astný et al. 2005). Sham-operated control rats (SHAM group) were i.c.v. injected with $0.25 \mu \mathrm{l}$ of saline. Selection of sham-operated or lesioned animals was made randomly (from each litter 3 pups were treated with neuroactive substance, 3 pups were treated with saline and 2 male pups remained as naïve). The pups were weaned on the PND 28 (the day of birth was denoted as PND 0). According to the type of brain lesion three groups of animals were used in this study: QUIN, NAAG and corresponding SHAM groups $(n=4-8$ animals per group in each pain test).

\section{Neuropathic pain model}

Persistent neuropathic pain in rats was evoked by chronic constriction of sciatic nerve (CCI) according to the model of Bennett and Xie (1988). For this test, young adult animals with neonatal brain damage (or sham-operated animals) were used on PND 50-55. Rats were anesthetized with pentobarbital $(40 \mathrm{mg} / \mathrm{kg}$ b.w., i.p.). Then the right sciatic nerve was exposed and, proximally to the trifurcation of the nerve, $5 \mathrm{~mm}$ of the sciatic nerve was freed from the adhering connective tissue. Around the loosened nerve four 4-0 chromic catgut sutures were loosely tied at intervals of approximately $1 \mathrm{~mm}$. Finally, the incisioned muscles and skin were sutured in layers.

\section{Acute nociceptive tests}

Plantar test and von Frey test were performed 2-5 days before the CCI and then 2-3 weeks after this operation. Formalin test was performed one week before the CCI. Each animal was used in only one nociceptive test; they were not repeatedly exposed to pain.

Pain threshold for the thermal stimulation was determined using plantar test equipment (Ugo Basile, Comerio, Italy) as described previously (Procházková et al. 2009). Shortly, in this test the latency (in seconds) of hindlimb withdrawal to the noxious thermal stimulation 
was measured. Each animal was placed individually in a clear plastic box with a clear glass floor and was allowed to acclimatize for $10 \mathrm{~min}$. Cut-off value was set to $22 \mathrm{~s}$ to prevent limb injury. The testing box was cleaned between each animal.

For the testing of mechanical allodynia and hyperalgesia calibrated nylon von Frey monofilaments (Touch-Test Sensory Evaluator, North Coast Medical Inc., Morgan Hill, USA) of different thicknesses were used as described elsewhere (Howard et al. 2005). With these filaments the plantar surface of hind limbs was exposed to varying degrees of pressure. The stimulus was applied five times at $2 \mathrm{~s}$ intervals for each successive filament until five clear withdrawal responses were elicited (100\% response).

For chemical nociception, formalin test (injection of $50 \mu \mathrm{l}$ of $2.5 \%$ formalin into the plantar surface of right hindpaw) was used. Scoring of nociceptive behavior began immediately after the formalin injection and continued for one hour. Nociceptive scores were determined for each 5-min time blocks using following behavioral categories: 0 - the injected paw is not favored; 1 - the injected paw has little or no weight placed on it; 2 - the injected paw is elevated and is not in contact with the surface; 3 - the injected paw is licked, bitten or shaken.

\section{Visualization of c-Fos positive neurons}

Two or three weeks after the CCI animals were deeply anesthetized with ketamine and perfused transcardially with saline followed by $4 \%$ formaldehyde. Lumbar laminectomy was performed and the $\mathrm{L}_{2-5}$ segments of the spinal cord were removed. Spinal cords were further fixed in $4 \%$ formaldehyde in $0.1 \mathrm{M}$ phosphate-buffered saline (PBS; $\mathrm{pH} 7.4$ ) for 2-3 days, and then soaked in $20 \%$ sucrose/PBS for cryoprotection. Lumbar spinal cords were cut into $50 \mu \mathrm{m}$ transversal section with a freezing microtome and treated as freefloating sections in cold PBS. The immunohistochemical protocol was similar to that given elsewhere (Yamazaki et al. 2001). Briefly, after several rinses in PBS, sections were treated with $0.3 \% \mathrm{H}_{2} \mathrm{O}_{2}$, rinsed in PBS and treated with normal blocking serum. Then the sections were incubated with a polyclonal c-Fos antibody (Santa Cruz Biotechnology, Inc., Santa Cruz, USA; diluted 1:4000) overnight. Sections were then rinsed with PBS and incubated with biotinylated secondary antibody and subsequently with the ABC complex (Vectastain Elite Kit, Vector). Neurons were visualized by incubation with diaminobenzidine and intensified with nickel solution (DAB Kit, Vector).

\section{Statistical analysis}

Statistical analysis was carried out by Origin 6 (Microcal Software, USA) and by Statistica 6.0 (StatSoft Inc., USA). Data are expressed as mean \pm S.E.M. Statistical differences were evaluated using paired Student's t-test for comparison of ipsilateral versus contralateral sides of the same experimental group. For comparison of the ipsilateral versus contralateral sides between groups one-way ANOVA test followed by posthoc Fisher's test were used. Differences between means were considered statistically significant if $p<0.05$. For the analysis of von Frey test the stimulus-response curves were constructed. The curves were fitted according to the equation $y=\left(y_{\max }-y_{\min }\right) /\left(1+\left(x / x_{0}\right)^{n}\right)+y_{\min }$. The value $x_{0}$ corresponds to the force eliciting $50 \%$ of the maximum response and was defined as threshold value. The thresholds were used in subsequent comparisons within tested groups.

\section{Results}

\section{Pain threshold for thermal stimulation}

Thresholds of plantar test in animals without neuropathic pain were significantly different in both schizophrenia model groups (QUIN and NAAG) compared to control group (SHAM). The thermal pain threshold in SHAM group (6.7 $\pm 0.7 \mathrm{~s})$ was significantly lower compared to groups neonatally treated with QUIN $(8.3 \pm 0.3 \mathrm{~s})$ and/or NAAG $(8.2 \pm 0.3 \mathrm{~s} ; \mathrm{p}<0.05)$ (Fig. 1). It means that the sensitivity to the thermal stimulation was attenuated by neonatal brain damage. Latencies were measured on right and left hindlimbs, but no lateralization of pain threshold was observed in any of the tested groups.

\section{Pain threshold for mechanical stimulation}

There were no differences between QUIN-, NAAG- and SHAM-operated groups using von Frey filaments (data not shown). On whole scale of filaments $(1 \mathrm{~g}-180 \mathrm{~g})$ we did not find any changes in the threshold for mechanical stimulation. It means that the neonatal brain lesion affected only the thermal hyperalgesia, but not the mechanical hyperalgesia (allodynia).

\section{Formalin test}

In early phase of the formalin test the acute 
chemical irritable pain was evaluated. Initially, we observed similar onset of the pain behavior in all three groups tested. This phase was shorter in QUIN-treated group compared to NAAG-treated group or controls (Figure 2). The data obtained in the late phase of formalin test (inflammatory pain) showed the more accented pain perception in animals with neonatal brain lesions compared to controls (Fig. 2). However, the differences did not reach statistical significance.

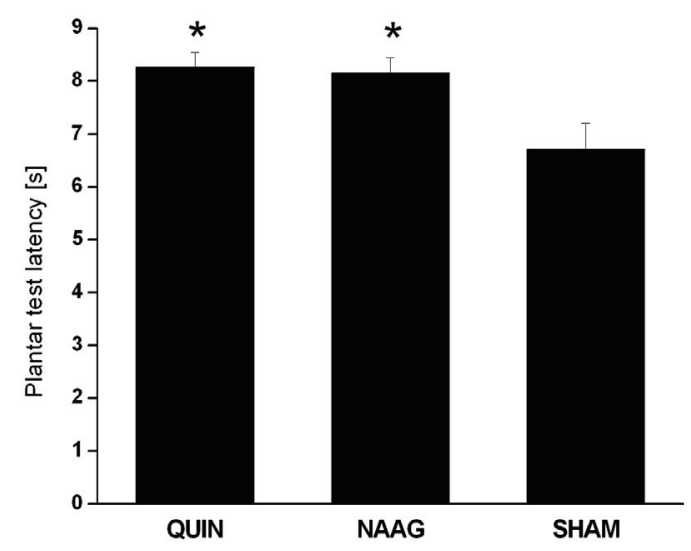

Fig. 1. Plantar test withdrawal latencies in rats without neuropathic pain. Both groups with neonatal brain lesion (QUIN and NAAG) show lower sensitivity to thermal stimulation compared to controls (SHAM). Latencies were measured on both hindlimbs. The height of each bar represents the mean time \pm S.E.M. of results obtained from $5-8$ animals. $* \mathrm{p}<0.05$ compared to SHAM group.

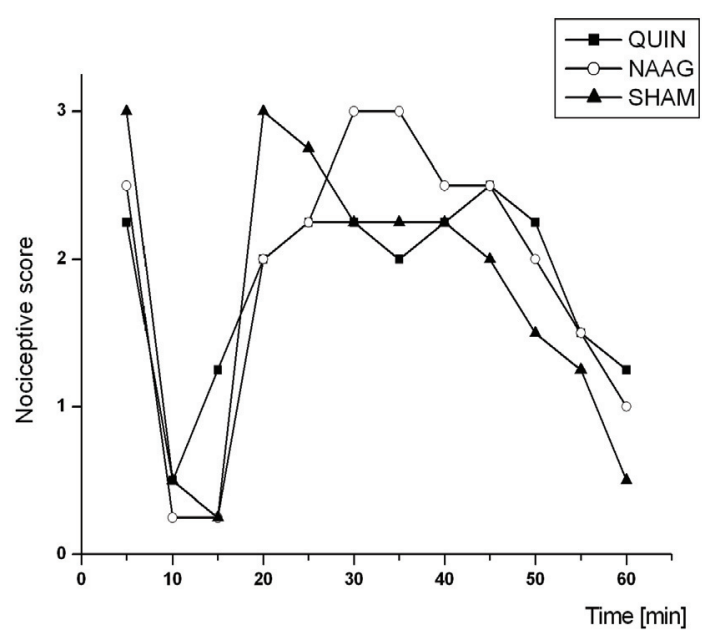

Fig. 2. Nociceptive responses induced by injection of $50 \mu \mathrm{l} 2.5 \%$ formalin in hind paw. Differences between QUIN $(n=8)$, NAAG $(n=5)$ and SHAM $(n=7)$ groups in any phase of formalin test were not significant.

\section{Neuropathic pain}

Chronic constriction of the sciatic nerve was used as the model of peripheral neuropathic pain. The operation was performed on adult animals (PND 50-55).
Two weeks after the operation the tests for mechanical and thermal allodynia/hyperalgesia were performed.

\section{Thermal nociception under the neuropathic pain}

As shown in Figure 3, the SHAM-operated group exhibited a significant decrease in the pain threshold on the ligated hind limb $(\mathrm{p}<0.05)$ compared to threshold latencies of pain perception before $(6.9 \pm 1.0 \mathrm{~s})$ and after the ligation $(3.7 \pm 1.2 \mathrm{~s})$. This difference is typical for the peripheral neuropathic pain and was not observed in either QUIN-treated (before ligation $8.4 \pm 0.3 \mathrm{~s}$, after ligation $8.0 \pm 1.3 \mathrm{~s}$ ) or NAAG-treated animals (before ligation $8.2 \pm 0.4 \mathrm{~s}$, after ligation $6.8 \pm 0.8 \mathrm{~s})$.

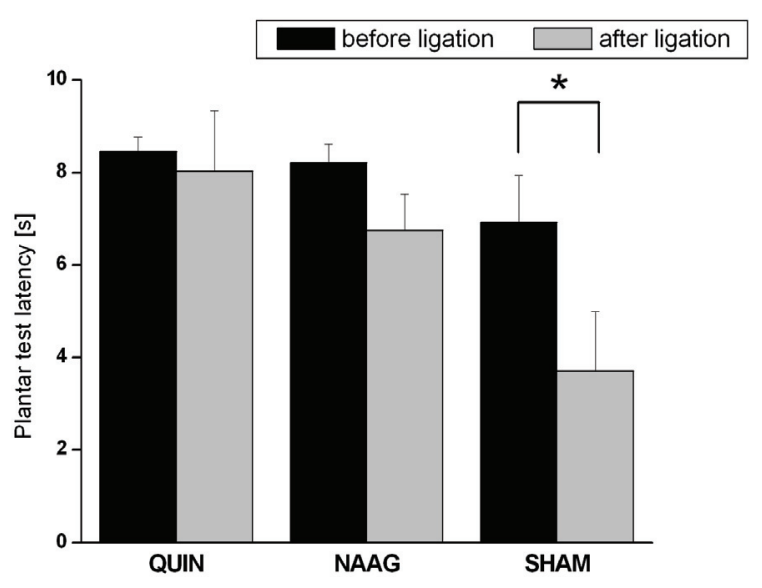

Fig. 3. Plantar test withdrawal latencies in rats 2-3 weeks after CCI. Significant difference before (dark bars) and after ligation (light bars) only in SHAM group was observed. The height of each bar represents the mean time \pm S.E.M. of results obtained from 5-7 animals. * $\mathrm{p}<0.05$.

\section{Mechanical nociception in neuropathic pain}

When von Frey filaments were used, the changes in mechanical allodynia/hyperalgesia were observed in all three tested groups with CCI. In QUIN-treated group the threshold value (log of $50 \%$ of maximum response) was significantly lower in ligated hindlimb $(0.76 \pm 0.22 \mathrm{log} g)$ comparing to contralateral side $(1.53 \pm 0.16$ log $\mathrm{g}$; Fig. 4A). Similar results were obtained in NAAG-treated (Fig. 4B; $0.99 \pm 0.24 \log \mathrm{g}$ and $1.65 \pm 0.12 \log \mathrm{g}$ ) on ipsilateral and contralateral side, respectively) and in SHAM-operated animals (Fig. 4C; $1.41 \pm 0.11 \mathrm{log} \mathrm{g}$ and $1.94 \pm 0.08 \log \mathrm{g}$ on ipsilateral and contralateral side, respectively). In both neonatally lesioned groups the perception of mechanical stimulation was higher in comparison with SHAM-operated animals (on both ipsilateral and contralateral sides), but only in QUINtreated group this effect reached statistical significance (Fig. 4D; $<<0.05$ ). 
A

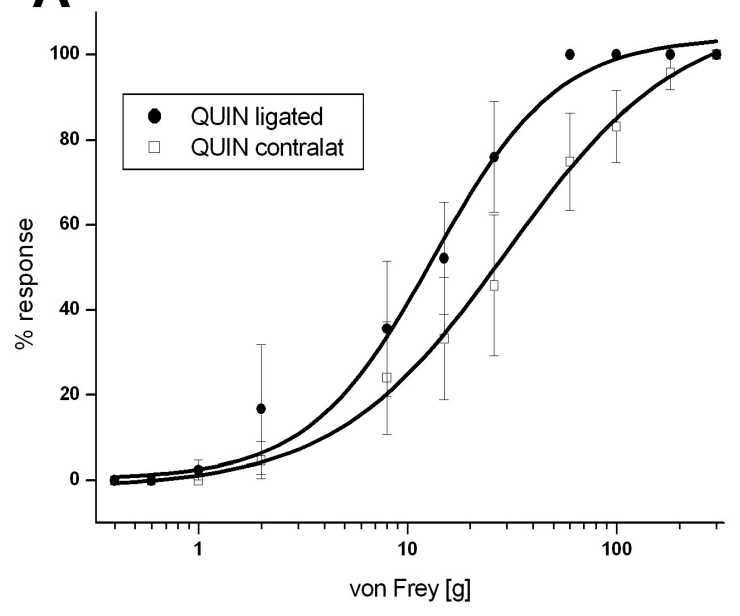

C

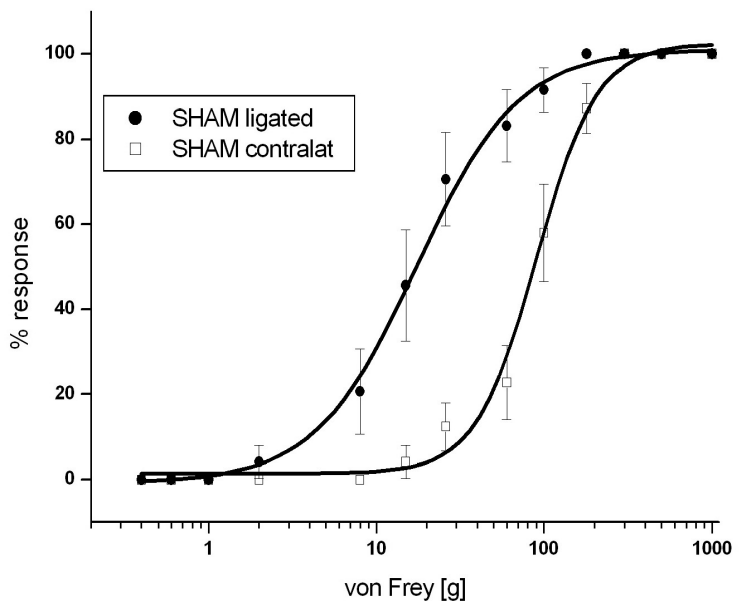

B

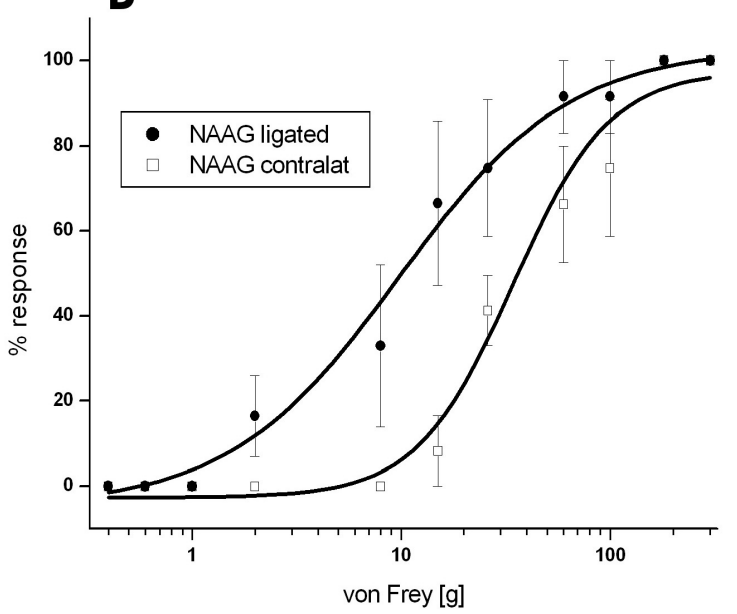

D

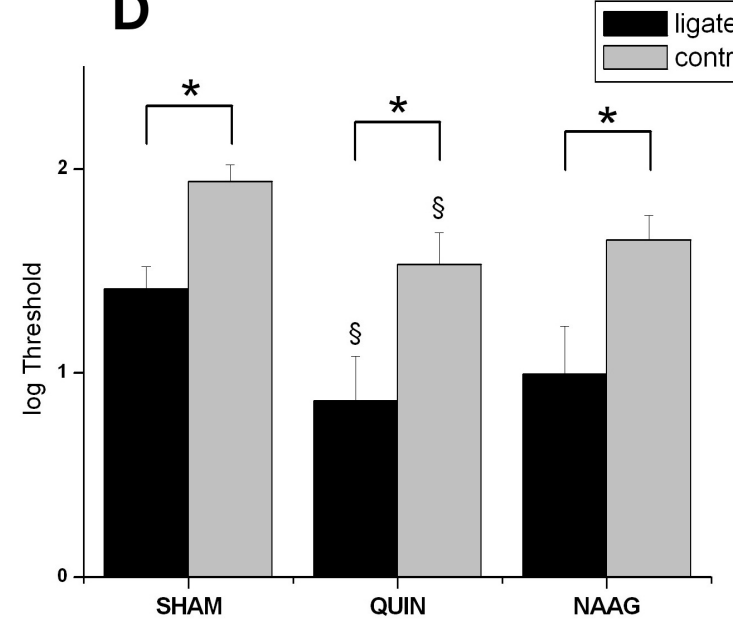

Fig. 4. Mechanical hyperalgesia/allodynia in rats $2-3$ weeks after CCI. Stimulus-response curves of ligated and contralateral hind limb for QUIN (A), NAAG (B) and SHAM (C) group. In all groups the threshold values (the force required to elicit $50 \%$ of the maximum response) on the ligated side (light bars) were significantly higher compared to unaffected side (dark bars) (D). In QUIN group both ligated and contralateral thresholds were lower compared to SHAM-operated animals. Values represent the mean \pm S.E.M. of results obtained from 5-7 animals. * $p<0.05,{ }^{\S} \mathrm{p}<0.05$ compared to SHAM group.

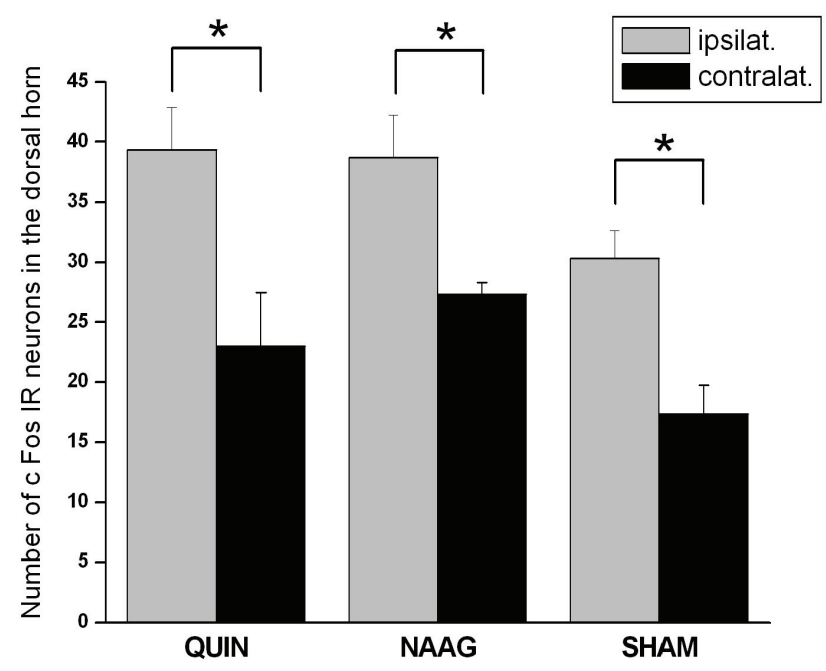

Fig. 5. Number of c-Fos-IR neurons in the spinal dorsal horn (segments L3-L5) in animals after CCI. In all groups the number of neurons on the ligated side (light bars) was significantly higher compared to unaffected side (dark bars), but there were no differences between QUIN, NAAG and SHAM groups. Each bar represents mean \pm S.E.M. from 5-7 animals. $* p<0.05$.

\section{c-Fos protein expression in neuropathic pain}

After nociceptive tests the rats with CCI were transcardially perfused and lumbar spinal segments were removed. Then c-Fos immunoreactive (IR) neurons in the spinal dorsal horn were visualized and their total number in this area was counted. The total number of c-Fos IR neurons was significantly higher on ligated side compared to the contralateral side (Fig. 5). This effect

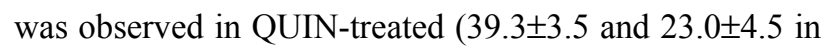


ipsilateral and contralateral dorsal horn, respectively; $\mathrm{p}<0.05)$, NAAG-treated $(38.7 \pm 3.6$ and $27.3 \pm 1.0$ in ipsilateral and contralateral dorsal horn, respectively; $\mathrm{p}<0.05)$ and SHAM-operated animals $(30.3 \pm 2.3$ and $17.4 \pm 2.4$ in ipsilateral and contralateral dorsal horn, respectively; $p<0.05$ ). The data suggest that there were no significant differences in the number of c-Fos IR neurons in animals with brain lesions in comparison with control group.

\section{Discussion}

In the present study we compared pain perception of two recently described neurodevelopmental models of schizophrenia based on the neurotoxicity of intracranially increased levels of endogenous NMDA-R agonists, QUIN and/or NAAG, in neonatal rat pups on PND 12. The weak agonists (Shave et al. 2001, Št'astný et al. 2005), with selective affinity to heteromers containing NR2B (QUIN) or NR2D subunits (NAAG), evoked nerve cell damage in periventricular rat brain region with a maximum in the hippocampus (Bubeníková-Valešová et al. 2006, Kudoh et al. 2000).

In the first model quinolinic acid, an endogenous metabolite of tryptophan with a partial selectivity for NR2B subunit containing NMDA-R (de Carvalho et al. 1996), was used. Although QUIN itself produced prolonged decrease in pain sensitivity in tail flick test (Heyliger et al. 1998), the pain-related changes observed in this study can be related to the decreased expression of NR2B protein in hippocampi of rats with the neonatal brain lesion (Skuba et al. 2004). The loss of the subunit protein seemed to be comparable to the action of subunit selective NMDA-R antagonists in alleviating chronic pain (Boyce et al. 1999, Chizh et al. 2001). In contrast to our results, adult rats with neonatal ventral hippocampal lesion exhibited decreased latencies for the thermal and mechanical nociception (Al Amin et al. 2004). Possible explanation of this difference can be in the contribution of the anterior cingulate cortex and amygdala in pain perception in adult rats (Gao et al. 2004). We assume that these brain regions can be damaged by QUIN infused intraventricularly, but not by ibotenic acid infused into the ventral hippocampus (Lipska et al. 1992).

In the case of the second model of schizophrenia, NAAG, an agonist at group II metabotropic glutamate receptors (mGluRs) (Neale et al. 2005), was infused to neonatal rat brains in the equimolar dose as in the case of QUIN. NAAG is one of the most abundant neurotransmiter in rat brain and is up to date the only known endogenous ligand of mGluR3. Its precise physiological function remains unknown. Previous reports have demonstrated that NAAG interacts not only at postsynaptic mGluRs, preferring subtype 3 to subtype 2 (Wroblewska et al. 1997), but at higher concentrations, also at NMDA-R heteromers (Losi et al. 2004, Shave et al. 2001), containing NR2D subunit. We previously showed that the interaction of NAAG with NMDA-Rs at high micromolar or low millimolar concentrations is responsible for neurotoxicity of this dipeptide in rat hippocampus (Bubeníková-Valešová et al. 2006, Pliss et al. 2000). NAAG-induced damage of the blood-brain barrier also seems to be involved in this process (Pliss et al. 2002). Moreover, elevated levels of extracellular NAAG, evoked by the inhibition of glutamatecarboxypeptidase II, produced an analgesic effect in animal pain models (Neale et al. 2005, Yamamoto et al. 2004).

The pain properties of these models were examined using followed pain tests: plantar test (acute thermal nociception), test with von Frey filaments (acute mechanical nociception) and formalin test (acute chemical irritable nociception and inflammatory nociception). For the chronic neuropathic pain the CCI model was used and following pain tests were performed in this model: plantar test (thermal hyperalgesia and allodynia), test with von Frey filaments (mechanical allodynia) and visualization of c-Fos positive neurons (spontaneous chronic pain). c-Fos protein is a marker of neuronal activity and its expression is evoked by various types of noxious stimulation (Coggeshall 2005).

We found the decreased pain perception in animals with both types of neurotoxic lesion only in thermal nociception test (plantar test). In other performed tests (mechanical nociception - von Frey filaments, chemical nociception - formalin test) the sensitivity to painful stimulation remained unchanged. These findings indicate that the neonatal brain lesion caused by QUIN or NAAG has only a limited influence on nociceptive pain perception in adulthood.

The CCI model appears to be one of the most frequently used models for the study of mechanism and treatment of neuropathic pain. Our findings demonstrating the presence of thermal and mechanical hyperalgesia/allodynia during two weeks after CCI are consistent with the definition of this model (Bennett and Xie 1988). We observed both types of pain in the control group, which corresponds with our previous study 
(Franěk et al. 2004). In the animals with neurodevelopmental models of schizophrenia the sensitivity to thermal and mechanical stimulation was different. While the pain threshold for thermal stimulation was not affected by CCI, in the pain threshold for mechanical stimulation we observed significant decrease in both SHAM and QUIN groups. Moreover, in the QUIN group the mechanical pain threshold was significantly lower than in control group. It means that the rats with described brain lesions are not sensitive to thermal hyperalgesia after CCI, but are sensitive to mechanical hyperalgesia/allodynia, even more than the control animals are. The difference in the development of thermal and mechanical pain perception in a model of neuropathic pain was described previously (Hofmann et al. 2003). Similar differences of thermal and mechanical pain after CCI were described in analgesic actions of some drugs (baclofen, amitryptiline) (De Vry et al. 2004). These facts indicate different mechanisms of thermal and mechanical hyperalgesia/allodynia in neuropathic pain. The symptoms of mechanical hyperalgesia/allodynia are considered to be more reliable than thermal hyperalgesia. This hypothesis is supported by more consistent pathophysiological mechanisms underlying the mechanical allodynia in different types of neuropathic pain (Bridges et al. 2001).

There are conflicting views about the correlation between numbers of c-Fos immunoreactive (IR) neurons in the spinal cord and severity of hyperalgesia/allodynia (Munglani et al. 1999, Yamazaki et al. 2001). In our study, the CCI significantly increases the number of ipsilateral c-Fos IR neurons compared to the number of contralateral ones in all groups. However, the increase of the neurons paralleled the development of both thermal and mechanical hyperalgesia/allodynia in sham-operated rats only. In QUIN and NAAG rats thermal hyperalgesia did not develop at all, so that the increase of the IR neurons rather followed the development of mechanical hyperalgesia/ allodynia. We could summarize that if there is a correlation, then it is between the number of c-Fos IR neurons and mechanical, but not thermal, hyperalgesia/ allodynia after CCI. We hypothesize that ipsilateral increase of c-Fos IR neurons might follow ongoing mechanical stimulation of the injured hindlimb during normal walking after CCI. Moreover, it supports the hypothesis about the significance of mechanical hyperalgesia/allodynia mentioned in previous paragraph.

We may conclude that the perception of thermal stimulation is attenuated in both neuropathic and nociceptive pain in described animal models based on the neonatal i.c.v infusion of QUIN or NAAG. Mechanical pain is exacerbated in neuropathic rats in both models, while the perception of acute chemical and inflammatory pain (formalin test) remains unchanged and comparable to controls. Thus, nociceptive and neuropathic pain belongs - in addition to behavioral changes and changes in social interactions - to the parameters which are affected in the neurodevelopmental animal models of schizophrenia. These findings highlight the importance of neonatal period in the development of pain pathways.

\section{Conflict of Interest}

There is no conflict of interest.

\section{Acknowledgements}

This work was supported by CNS 1M0517, by Research Goal 21620816 and by GACR 304/10/1274.

\section{References}

AL AMIN HA, ATWEH SF, JABBUR SJ, SAADÉ NE: Effects of ventral hippocampal lesion on thermal and mechanical nociception in neonates and adult rats. Eur J Neurosci 20: 3027-3034, 2004.

BENNETT GJ, XIE YK: A peripheral mononeuropathy in rat that produces disorders of pain sensation like those seen in man. Pain 33: 87-107, 1988.

BLUMENSOHN R, RINGLER D, ELI I: Pain perception in patients with schizophrenia. J Nerv Ment Dis 190: 481483, 2002.

BOYCE S, WYATT A, WEBB JK, O'DONNELL R, MASON G, RIGBY M, SIRINATHSINGHJI D, HILL RG, RUPNIAK NM: Selective NMDA NR2B antagonists induce antinociception without motor dysfunction: correlation with restricted localisation of NR2B subunit in dorsal horn. Neuropharmacology 38: 611-623, 1999.

BRIDGES D, THOMPSON SW, RICE AS: Mechanisms of neuropathic pain. Br J Anaesth 87: 12-26, 2001. 
BUBENÍKOVÁ-VALEŠOVÁ V, BALCAR VJ, TEJKALOVÁ H, LANGMEIER M, ŠŤASTNÝ F: Neonatal administration of N-acetyl-L-aspartyl-L-glutamate induces early neurodegeneration in hippocampus and alters behaviour in young adult rats. Neurochem Int 48: 515-522, 2006.

CHIZH BA, HEADLEY PM, TZSCHENTKE TM: NMDA receptor antagonists as analgesics: focus on the NR2B subtype. Trends Pharmacol Sci 22: 636-642, 2001.

COGGESHALL RE: Fos, nociception and the dorsal horn. Prog Neurobiol 77: 299-352, 2005.

DE CARVALHO LP, BOCHET P, ROSSIER J: The endogenous agonist quinolinic acid and the non endogenous homoquinolinic acid discriminate between NMDAR2 receptor subunits. Neurochem Int 28: 445-452, 1996.

DE VRY J, KUHL E, FRANKEN-KUNKEL P, ECKEL G: Pharmacological characterization of the chronic constriction injury model of neuropathic pain. Eur J Pharmacol 491: 137-148, 2004.

DUNCAN GE, MOY SS, PEREZ A, EDDY DM, ZINZOW WM, LIEBERMAN JA, SNOUWAERT JN, KOLLER BH: Deficits in sensorimotor gating and tests of social behavior in a genetic model of reduced NMDA receptor function. Behav Brain Res 153: 507-519, 2004.

DWORKIN RH: Pain insensitivity in schizophrenia: a neglected phenomenon and some implications. Schizophr Bull 20: 235-248, 1994.

FISHBAIN DA: Pain insensitivity in psychosis. Ann Emerg Med 11: 630-632, 1982.

FRANĚK M, VACULÍN S, ROKYTA R: GABA $\mathrm{B}_{\mathrm{B}}$ receptor agonist baclofen has non-specific antinociceptive effect in the model of peripheral neuropathy in the rat. Physiol Res 53: 351-355, 2004.

GAO YJ, REN WH, ZHANG YQ, ZHAO ZQ: Contributions of the anterior cingulate cortex and amygdala to pain- and fear-conditioned place avoidance in rats. Pain 110: 343-353, 2004.

HEYES MP, ACHIM CL, WILEY CA, MAJOR EO, SAITO K, MARKEY SP: Human microglia convert L-tryptophan into the neurotoxin quinolinic acid. Biochem J 320: 595-597, 1996.

HEYLIGER SO, GOODMAN CB, NGONG JM, SOLIMAN KF: The analgesic effects of tryptophan and its metabolites in the rat. Pharmacol Res 38: 243-250, 1998.

HOFMANN HA, DE VRY J, SIEGLING A, SPREYER P, DENZER D: Pharmacological sensitivity and gene expression analysis of the tibial nerve injury model of neuropathic pain. Eur J Pharmacol 470: 17-25, 2003.

HOOLEY JM, DELGADO ML: Pain insensitivity in the relatives of schizophrenia patients. Schizophr Res 47: 265-273, 2001.

HOWARD RF, WALKER SM, MICHAEL MP, FITZGERALD M: The ontogeny of neuropathic pain: Postnatal onset of mechanical allodynia in rat spared nerve injury (SNI) and chronic constriction injury (CCI) models. Pain 115: 382-389, 2005.

KUDOH A, ISHIHARA H, MATSUKI A: Current perception thresholds and postoperative pain in schizophrenic patients. Reg Anesth Pain Med 25: 475-479, 2000.

LIPSKA BK: Using animal models to test a neurodevelopmental hypothesis of schizophrenia. $J$ Psychiatry Neurosci 29: 282-286, 2004.

LIPSKA BK, JASKIW GE, CHRAPUSTA S, KAROUM F, WEINBERGER DR: Ibotenic acid lesion of the ventral hippocampus differentially affects dopamine and its metabolites in the nucleus accumbens and prefrontal cortex in the rat. Brain Res 585: 1-6, 1992.

LISÝ V, DVOŘÁKOVÁ L, ŠŤASTNÝ F: Altered glutamate binding following quinolinate lesions in developing rat brain. Exp Neurol 125: 82-86, 1994.

LOSI G, VICINI S, NEALE J: NAAG fails to antagonize synaptic and extrasynaptic NMDA receptors in cerebellar granule neurons. Neuropharmacology 46: 490-496, 2004.

MARCOTTE ER, PEARSON DM, SRIVASTAVA LK: Animal models of schizophrenia: a critical review. J Psychiatry Neurosci 26: 395-410, 2001.

MUNGLANI R, HUDSPITH MJ, FLEMING B, HARRISSON S, SMITH G, BOUNTRA C, ELLIOT PJ, BIRCH PJ, HUNT SP: Effect of pre-emptive NMDA antagonist treatment on long-term Fos expression and hyperalgesia in a model of chronic neuropathic pain. Brain Res 822: 210-219, 1999.

NEALE JH, OLSZEWSKI RT, GEHL LM, WROBLEWSKA B, BZDEGA T: The neurotransmitter Nacetylaspartylglutamate in models of pain, ALS, diabetic neuropathy, CNS injury and schizophrenia. Trends Pharmacol Sci 26: 477-484, 2005. 
PASSANI L, ELKABES S, COYLE JT: Evidence for the presence of N-acetylaspartylglutamate in cultured oligodendrocytes and LPS activated microglia. Brain Res 794: 143-145, 1998.

PLISS L, FITZGIBBON T, BALCAR VJ, ŠŤASTNÝ F: Neurotoxicity of NAAG in vivo is sensitive to NMDA antagonists and mGluR II ligands. Neuroreport 11: 3651-3654, 2000.

PLISS L, JEŽOVÁ D, MAREŠ V, BALCAR VJ, ŠŤASTNÝ F: N-Acetyl-L-aspartyl-L-glutamate changes functional and structural properties of rat blood-brain barrier. Neurosci Lett 317: 85-88, 2002.

PROCHÁZKOVÁ M, ZANVIT P, DOLEŽAL T, PROKEŠOVÁ L, KRŠIAK M: Increased gene expression and production of spinal cyclooxygenase 1 and 2 during experimental osteoarthritis pain. Physiol Res 58: 419-425, 2009.

ROSENTHAL SH, PORTER KA, COFFEY B: Pain insensitivity in schizophrenia. Case report and review of the literature. Gen Hosp Psychiatry 12: 319-322, 1990.

SCHNEIDER T, PRZEWLOCKI R: Behavioral alterations in rats prenatally exposed to valproic acid: animal model of autism. Neuropsychopharmacology 30: 80-89, 2005.

SHAVE E, PLISS L, LAWRANCE ML, FITZGIBBON T, ŠŤASTNÝ F, BALCAR VJ: Regional distribution and pharmacological characteristics of $\left[{ }^{3} \mathrm{H}\right] \mathrm{N}$-acetyl-aspartyl-glutamate (NAAG) binding sites in rat brain. Neurochem Int 38: 53-62, 2001.

SKUBA I, TEJKALOVÁ H, ŠŤASTNÝ F: Changes in subunit composition of N-methyl-D-aspartate receptor in animal model of schizophrenia: effect of subunit selective antagonist. Eur Neuropsychopharmacol 14 (Suppl 1): S73S74, 2004.

ŠŤASTNÝ F, TEJKALOVA H, SKUBA I, BALCAR VJ, KAISER M, YONEDA Y: Quinolinic acid, N-methyl-Daspartate receptor and schizophrenia: testing an integrative theory. In: Amino Acid Signaling 04. YONEDA Y (ed), Research Signpost, Kerala, 2005, pp 67-83.

WROBLEWSKA B, WROBLEWSKI JT, PSHENICHKIN S, SURIN A, SULLIVAN SE, NEALE JH: $\mathrm{N}$-acetylaspartylglutamate selectively activates mGluR3 receptors in transfected cells. J Neurochem 69: 174181, 1997.

YAMAMOTO T, HIRASAWA S, WROBLEWSKA B, GRAJKOWSKA E, ZHOU J, KOZIKOWSKI A, WROBLEWSKI J, NEALE JH: Antinociceptive effects of N-acetylaspartylglutamate (NAAG) peptidase inhibitors ZJ-11, ZJ-17 and ZJ-43 in the rat formalin test and in the rat neuropathic pain model. Eur J Neurosci 20: 483-494, 2004.

YAMAZAKI Y, MAEDA T, SOMEYA G, WAKISAKA S: Temporal and spatial distribution of Fos protein in the lumbar spinal dorsal horn neurons in the rat with chronic constriction injury to the sciatic nerve. Brain Res 914: 106-114, 2001.

ZIMMERMANN M: Ethical guidelines for investigations of experimental pain in conscious animals. Pain 16: 109-110, 1983. 